are $\frac{1}{2}\left(-\sin ^{3} A+\sin ^{2} B+\sin ^{3} C\right), \quad \frac{1}{2}\left(\sin ^{3} A-\sin ^{3} B+\sin ^{2} C\right)$, $\frac{1}{2}\left(\sin ^{2} A+\sin ^{2} B-\sin ^{2} C\right)$ respectively. But we have

and thence

$$
A+B+C=\pi
$$

$$
\begin{aligned}
& \sin ^{2} A+\sin ^{2} B-\sin ^{2} C, \\
= & \sin ^{2} A+\sin ^{2} B-\sin ^{2}(A+B) \\
= & 2 \sin A \sin B(\sin A \sin B-\cos A \cos B), \\
= & -2 \sin A \sin B \cos (A+B), \\
= & 2 \sin A \sin B \cos C,
\end{aligned}
$$

and we thus have

$a, b, c=\sin B \sin C \cos A, \sin C \sin A \cos B, \sin A \sin B \cos C$, (or, what is the same thing, $a: b: c=\cot A: \cot B: \cot C$ ), and the equation of the circle is

$y z \sin ^{2} A+z x \sin ^{2} B+x y \sin ^{2} C$

$-\frac{1}{2}(x \sin B \sin C \cos A+y \sin C \sin A \cos B+z \sin A \sin B \cos C)$

$$
\times(x+y+z)=0 .
$$

We thus have $x: y: z=1: 1: 1$ for the point $O^{\prime}$, and $x: y: z=\cot A: \cot B: \cot C$ for the point $O ; \operatorname{viz} O^{\prime}$ is the point of intersection of the lines from the angles to the midpoints of the opposite sides respectively; and $O$ is the point of intersection of the perpendiculars from the angles on the opposite sides respectively: and the foregoing equation is consequently that of the Nine-points Circle.

\title{
ON THE NINE-POINTS CIRCLE OF A PLANE TRIANGLE.
}

\section{By Professor Cayley.}

I CONSIDER the circle which meets the sides of a triangle $A B C$ in the points $F, L ; G, M ; H, N$ respectively, where ultimately $F, G, H$ are the feet of the perpendiculars let fall from the angles on the opposite sides, and $L, M, N$ are the wid-points of the sides: but in the first instance they are 
taken to be arbitrary points. Taking the radius of the circle to be unity, the coordinates of the point $F$ may be taken to be $\cos F, \sin F$, and these may be expressed rationally in terms of the tangent of the half-angle, $f=\tan \frac{1}{2} F$; and similarly for the other points, viz. we may determine the six points by the parameters $f, g, h, l, m, n$ respectively. The sides of the triangle are the lines joining the points $L, F$; $M, G ; N, H$ respectively: thus the equations of the sides are

$$
\begin{aligned}
& \text { for } B C x(1-l f)+y(l+f)-(1+l f)=0 \text {, say } U=0 \text {, }
\end{aligned}
$$

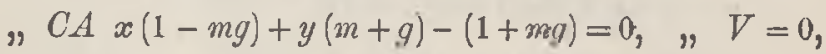

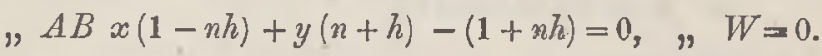

We have $A F$ a line through the intersections of $B C$ and $C A$; its equation is therefore of the form $B V-C W=0$, and to determine $B, C$ we have $B V_{0}-C W_{0}=0$, if $V_{*}, W_{0}$ are the values of $V, W$ belonging to the point $F$, the coordinates of which are $\frac{1-f^{2}}{1+f^{2}}, \frac{2 f}{1+f^{2}}$; we find

$$
\begin{aligned}
& V_{0}=-2(f-g)(f-m) \div\left(1+f^{2}\right) ; \\
& W_{0}=2(h-f)(f-n) \div\left(1+f^{3}\right),
\end{aligned}
$$

and then $B \div C=W_{0} \div V_{0}$ : we thus find

equation

$$
\begin{aligned}
& A F \text { is } B V-C W=0, \\
& B C, C^{\prime} W-A^{\prime} U=0, \\
& C I I, A^{\prime \prime} U-B^{\prime \prime} V=0 .
\end{aligned}
$$

where $B: C=-(h-f)(f-n):(f-g)(f-m)$,

$$
\begin{aligned}
& C^{\prime}: A^{\prime}=-(f-g)(g-l):(g-h)(g-n), \\
& A^{\prime \prime}: B^{\prime \prime}=-(g-h)(h-m):(h-f)(h-l) .
\end{aligned}
$$

The condition in order that the three lines may meet in a point is $C B^{\prime} A^{\prime \prime}=C A^{\prime} B^{\prime \prime \prime}$, viz, this is

$$
(f-n)(g-l)(h-m)+(f-m)(g-n)(h-l)=0,
$$

or, as this may also be written,

$2 f g h-g h(m+n)-h f(n+l)-f g(l+m)$

$$
+m n(g+h)+n l(h+f)+l m(f+g)-2 \ln n=0,
$$


Similarly

equation

$$
\begin{aligned}
& A L \text { is } \mathbf{B} V-\mathfrak{C} W=0, \\
& B M, \mathbb{C}^{\prime} W-\mathfrak{A}^{\prime} U=0, \\
& C N, \mathfrak{A}^{\prime \prime} U-\mathfrak{B}^{\prime \prime} V=0 .
\end{aligned}
$$

where

$$
\begin{aligned}
& 13: \mathfrak{C}=-(n-l)(h-l):(l-m)(g-l), \\
& \mathfrak{C}^{\prime}: \mathfrak{A}^{\prime}=-(l-m)(f-m):(m-n)(h-m), \\
& \mathfrak{A}^{\prime \prime}: \mathbf{b}^{\prime \prime}=-(m+n)(g-n):(n-l)(f-n),
\end{aligned}
$$

and the condition in order that the three lines may meet in a point is $\mathbf{B C} \mathfrak{C}^{\prime \prime}=\mathfrak{C} \mathfrak{A} \mathbf{A B}^{\prime \prime}$, viz. this is the same condition as before; that is if the lines $A F, B G, C H$ meet in a point, then also the lines $A L, B M, C N$ will meet in a point.

In the case of the nine-points circle we have $M N, N L$, $L M$ parallel to $L F, M G, N H$ respectively: the equation of $M N$ is

$$
x(l-m n)+y(m+n)-(l+m n)=0,
$$

and this is parallel to $L F$, if

$$
\frac{m+n}{1-m n}=\frac{l+f}{1-l f}, \text { that is } L+F=M+N \text {. }
$$

Hence for the nine-points circle we have

$$
L+F=M+N, M+G=N+L, N+H=L+M,
$$

or, as these equations may be written,

$$
2 L=G+H, 2 M=H+F, 2 N=F+G,
$$

viz. it thus appears that the radii to the points $L, M, N$ respectively, or say the radii $L . M . N$, bisect the angles usade by the radii $G$ and $I, I I$ and $F, F$ and $G$ respectively.

It may be added that we have

$$
\begin{aligned}
& m+n-l+l m n=f\{1-m n+l(m+n)\}, \\
& n+l-m+l m n=g\{1-n l+m(n+l)\}, \\
& l+m-n+l m n=h\{1-l m+n(l+m)\},
\end{aligned}
$$

viz. $f, g, h$ are expressible each of them as a rational function of $l, m, n$. 